\title{
Cholecystectomy and duodenogastric reflux: interacting effects over the gastric mucosa
}

\author{
Erdinc Mercan ${ }^{1}$, Ugur Duman ${ }^{1}$, Deniz Tihan ${ }^{1}$, Evren Dilektasli ${ }^{1}$ and Kazim Senol ${ }^{1,2^{*}}$
}

\begin{abstract}
Aims: To evaluate association between duodenogastric reflux and early gastric mucosal changes before and after the cholecystectomy procedure.

Materials and methods: Patients were evaluated with preoperative and postoperative endoscopy and endoscopic biopsy. Demographic and clinical characteristics, histological parameters, presence of duodenogastric reflux, and Updated Sydney scores were noted.

Results: A total of fifty patients who obeyed the follow-up were enrolled into the study. Median age of the patients was 43 years (range 25-84). Male-female ratio was 0.51 (17/33). Duodenogastric reflux \% and Updated Sydney scores before and after cholecystectomy were $24(48 \%)$ versus $39(78 \%)$ and $2.38 \pm 2.21$ versus $3.46 \pm 3.05$, respectively $(p=0.001, p<0.000)$. Mucosal inflammation degree showed significant increase in $15(30 \%)$ patients, decrease in 7 (14\%) patients and equality in $28(56 \%)$ patients ( $p=0.037$ ). Neutrophil activation degree was significantly higher in $21(42 \%)$ patients, lower in $5(10 \%)$ patients after the surgery $(p=0.005)$. Postoperative glandular atrophy degree was also higher in $13(26 \%)$ patients and equal in $37(74 \%)$ patients $(p=0.001)$. Pre- and postoperative degree of intestinal metaplasia and $H$. pylori density did not any show significant difference $(p=0.157, p=0.248$, respectively). There were significant positive correlation between postoperative H. pylori infection and mucosal activity, inflammation, atrophy and intestinal metaplasia.
\end{abstract}

Conclusion: Cholecystectomy is a potent inducer of pathologic duodenogastric reflux. Early onset of duodenogastric reflux and underlying $H$. pylori gastritis cause early gastric mucosal injury following cholecystectomy procedure by interacting collectively.

Keywords: Laparoscopic cholecystectomy, Reflux gastritis, H. pylori

\section{Background}

Cholelitiasis is one of the most common problems affecting the digestive tract. Although the incidence depends on the factors including age, gender and ethnicity, $10-15 \%$ of the adult population has the gall bladder stones (Schafer et al. 1998). Therefore, cholecystectomy procedure is the second most practiced surgical procedure.

\footnotetext{
*Correspondence: kazimsenol@gmail.com; kazimsenol@hotmail.com 2 Department of General Surgery, Bursa State Hospital, Bursa, Turkey Full list of author information is available at the end of the article
}

Majority of the symptoms resolve after the cholecystectomy procedure. However, $15-20 \%$ of the patients present with new gastrointestinal symptoms or have continuous preoperative complaints (Manifold et al. 2000). Following the cholecystectomy procedure, the loss of reservoir function of gall bladder causes impairment in cyclic pattern of bile juice excretion; the loss of neurohumoral responds also causes motility changes in upper gastrointestinal system and should lead to increased duodenogastric reflux (DGR) (Perdikis et al. 1994). Thus, regurgitation of duodenal contents into the stomach induces the gastric mucosal injury resulting in persistent 
symptoms such as epigastric pain, nausea and bilious vomiting (Buxbaum 1982; Brough et al. 1984).

Pathologic DGR is observed in $51-89 \%$ of the patients after the cholecystectomy procedure (Fall et al. 2007; Chen et al. 2010). During the postoperative period, it has been demonstrated that bile refluxate levels and DGR severity increase in significant correlation with progressive atrophic gastritis (Lorusso et al. 1992). To reveal the underlying causes of gastric mucosal changes regarding post-cholecystectomy DGR, patients are evaluated with endoscopic examination and histological sampling. Reflux Gastritis Score (RGS) (Dixon et al. 1986) and Biliary Reflux Index (BRI) (Dixon et al. 2001) scoring systems have been established to identify the degree of mucosal injury and reactive gastritis. However, RGS and BRI scoring systems are unable to demonstrate the atrophic gastric mucosal changes due to long-term exposure of bile refluxate. Updated Sydney scoring system has the superiority over these scoring systems by providing the glandular atrophy and intestinal metaplasia degree (Dixon et al. 1996). In the literature, there are few studies attempted to evaluate the post-cholecystectomy DGR and mucosal atrophy related to DGR with Updated Sydney scoring system (Lin et al. 2009; Kellosalo et al. 1991; Zullo et al. 1998).

In this prospective observational cohort study, we aimed to identify the DGR and early atrophic changes of the gastric mucosa before and after cholecystectomy procedure with Updated Sydney scoring system.

\section{Methods}

This prospective cohort study was conducted in a referral gastrointestinal endoscopy unit of Bursa Yuksek Ihtisas Training and Research Hospital between January 2012 and March 2013. Institutional ethics committee approved the clinical research with 10,877 approval code. All patients provided written informed consent. Patients requiring cholecystectomy procedure due to symptomatic gallstone disease (episodic pain in epigastrium and right upper quadrant, bloating and belching associated with the pain vs.) were enrolled into the study. All of the patients were evaluated with Esophagogastroduodenoscopy (EGD) and endoscopic biopsy 30 days before the surgery. Postoperative EGD was performed to display the improvements of duodenogastric reflux (DGR) and histological alterations 60 days after the surgery. Observation of active bile reflux, presence of bilious gastric lake and gastritis were noted. Antral biopsies were taken $2-3 \mathrm{~cm}$ over the pylorus along the lesser and greater curvature. The specimens were placed in a $10 \%$ formalin solution, embedded in paraffin blocks and stained with hematoxylin/eosin Alcian-blue (AB, pH 2.5)/periodic acid Schiff (PAS). Histological parameters including chronic inflammation, neutrophil activation, glandular atrophy, intestinal metaplasia and presence of $H$. pylori were evaluated. Gastric mucosal changes and microbiological aspects of the chronic gastritis were reviewed with Updated Sydney classification system and graded into absent, mild, moderate and severe disease. Demographic data and clinical parameters of the patients were also recorded.

Patients with chronic co-morbid diseases (diabetes mellitus, coronary artery disease, and hypertension), long-term non-steroidal analgesic and oral contraceptive drug use, previous history of biliary and/or gastric surgery, gastric malignancy and ulcer, cholecystectomy during pregnancy, conversion to open surgery were excluded from the study.

Early morphologic changes of gastric mucosa and evidence of enterogastric reflux after laparoscopic cholecystectomy was determined as the main clinical outcome of this study.

\section{Statistical analyses}

Shapiro-Wilk test were used to assess normality. The normally distributed data was presented as mean $\pm \mathrm{SD}$ (standard deviation) and non-normally distributed data was presented as median value (interquartile range). Baseline characteristics were compared with paired Student's $t$ test or the Wilcoxon Signed-rank test for continuous variables or Mcnemar's test for categorical variables. The Wilcoxon signed-rank test was also used to assess differences before and after the surgery in each group. The Spearman's rank correlation was used to define a correlation between histological parameters and DGR. All statistical procedures were performed with SPSS 15.0 (SPSS Inc, Chicago, Illinois). $\mathrm{p}<0.05$ was considered as significant.

\section{Results}

50 patients who evaluated with pre- and post-cholecystectomy endoscopy and attended follow-up regularly were enrolled into the study. Median age of the patients was 43 years (range 25-84). Male-female ratio was 0.51 (17/33). Cholecystectomy etiology was stones in all patients. Mean time of preoperative esophagogastroduodenoscopy (EGD) and endoscopic biopsy were $38 \pm 8$ days. Preoperative endoscopy revealed duodenogastric reflux (DGR) in 24 (48\%) patients and H. pylori infection in $14(28 \%)$ patients. Updated Sydney score mean value before surgery was $2.38 \pm 2.21$ (range $0-11$ ). Demographic and clinical characteristics of patients were demonstrated in Table 1. Laparoscopic cholecystectomy was successfully performed in all patients without any complication. Postoperative follow-up was uneventful and all patients were discharged on postoperative first day. Mean time of postoperative EGD and endoscopic 


\begin{tabular}{|c|c|c|c|}
\hline & & Variables & $\mathrm{p}$ value \\
\hline Age (years) & & $43(25-84)$ & \\
\hline Gender, M/F & & $0.51(17 / 33)$ & \\
\hline \multirow[t]{2}{*}{ EGD, mean time (days) } & Preoperative & $38 \pm 8$ & \\
\hline & Postoperative & $56 \pm 10$ & \\
\hline \multirow[t]{2}{*}{ Presence of DGR $(n, \%)$} & Preoperative & $24(48 \%)$ & $0.001^{*}$ \\
\hline & Postoperative & $39(78 \%)$ & \\
\hline \multirow{2}{*}{$\begin{array}{l}\text { Presence of H. pylori infection } \\
(\mathrm{n}, \%)\end{array}$} & Preoperative & $14(28 \%)$ & $0.388^{*}$ \\
\hline & Postoperative & $18(36 \%)$ & \\
\hline \multirow[t]{2}{*}{ Sydney Score, mean value } & Preoperative & $2.38 \pm 2.21$ & $0.001^{* *}$ \\
\hline & Postoperative & $3.46 \pm 3.05$ & \\
\hline
\end{tabular}

${ }^{*}$ Mcnemar's test; ** Wilcoxon signed-rank test

biopsy were $56 \pm 10$ days. Postoperative EGD revealed significantly higher DGR in $39(78 \%)$ patients $(\mathrm{p}=0.001)$. Pre- and post-operative changes in lymphocytes and plasma cells degree, polymorph infiltration of neutrophil in the lamina propria, loss of specialized glands from either antrum or body, intestinal metaplasia of the foveolar or surface epithelium and density of $H$. pylori overlying epithelium rates and statistical significances were demonstrated in Table 2.

Postoperative pathological samples revealed that degree of inflammation, neutrophil activation, and glandular atrophy was significantly higher than the preoperative results. Chronic inflammation degree significantly showed an increase in $15(30 \%)$ patients, decrease in $7(14 \%)$ patients and remained as the same in $8(16 \%)$ patients after laparoscopic cholecystectomy procedure $(\mathrm{p}=0.007)$. Neutrophil activation degree was significantly higher in 21 (42\%) patients, lower in $5(10 \%)$ patients after the surgery $(\mathrm{p}=0.005)$. Postoperative glandular atrophy degree was also higher in 13 (26\%) patients and equal in $37(74 \%)$ patients $(\mathrm{p}=0.001)$. Pre- and postoperative degree of intestinal metaplasia and $H$. pylori density did not any show significant difference $(\mathrm{p}=0.157$, $\mathrm{p}=0.248$, respectively). Postoperative $H$. pylori infection was reported in 18 (36\%) patients. Pre- and post-operative degree of $H$. pylori density was assessed and graded in moderate and severe in $4 \%$ each, and mild in $16,24 \%$ of a total of 28 and $36 \%$ cases with atrophic changes, respectively. Postoperative $H$. pylori infection presented positive correlation with postoperative gastric mucosal changes including chronic inflammation $(r=0.41$, $\mathrm{p}=0.003)$, neuthrophil activation $(\mathrm{r}=0.49, \mathrm{p}=0.001)$, glandular atrophy $(\mathrm{r}=0.30, \mathrm{p}=0.03)$, intestinal metaplasia $(\mathrm{r}=0.48, \mathrm{p}=0.001)$. However, postoperative $H$. pylori infection did not show significant correlation with postoperative DGR $(r=0.08, p=0.55)$. In regard with these findings, postoperative Updated Sydney score mean value was significantly increased to $3.46 \pm 3.05$ (range $0-12, \mathrm{p}<0.000$ ). However pre- and post-operative severity of gastric inflammation according to Updated Sydney score classification was not significant in between mild, moderate and severe grades of disease $(\mathrm{p}=0.109)$.

\section{Discussion}

DGR is physiological in humans (Ritchie 1984). Thus, reflux gastritis is found to be associated with the amount of bile refluxate. Antral biopsies revealed decreased and even no gastritis in patients with post-cholecystectomy DGR (Farsakh et al. 1995). In contrast, Wilson et al. have concluded that excessive DGR after cholecystectomy is found to be associated with persistent

Table 2 Pre- and post-operative endoscopic and histological inflammation scores

\begin{tabular}{|c|c|c|c|c|c|c|c|c|c|c|}
\hline & & \multicolumn{2}{|c|}{ Mild } & \multicolumn{2}{|c|}{ Moderate } & \multicolumn{2}{|c|}{ Severe } & \multicolumn{2}{|c|}{ Total } & \multirow[t]{2}{*}{$p$ value } \\
\hline & & $\mathrm{n}$ & $\%$ & $\mathrm{n}$ & $\%$ & $\mathbf{n}$ & $\%$ & $\mathrm{n}$ & $\%$ & \\
\hline \multirow[t]{2}{*}{ Inflammation } & Preoperative & 15 & $(30 \%)$ & 3 & $(6 \%)$ & 1 & $(2 \%)$ & 19 & $(38 \%)$ & 0.037 \\
\hline & Postoperative & 15 & $(30 \%)$ & 6 & $(12 \%)$ & 3 & $(6 \%)$ & 24 & $(48 \%)$ & \\
\hline \multirow[t]{2}{*}{ Activity } & Preoperative & 12 & $(24 \%)$ & 6 & $(12 \%)$ & 1 & $(2 \%)$ & 19 & $(38 \%)$ & 0.005 \\
\hline & Postoperative & 22 & $(44 \%)$ & 8 & $(16 \%)$ & 2 & $(4 \%)$ & 32 & $(64 \%)$ & \\
\hline \multirow[t]{2}{*}{ Atrophy } & Preoperative & 14 & $(28 \%)$ & 3 & $(6 \%)$ & - & $(-\%)$ & 17 & $(34 \%)$ & 0.001 \\
\hline & Postoperative & 13 & $(26 \%)$ & 9 & $(18 \%)$ & 2 & $(4 \%)$ & 24 & $(48 \%)$ & \\
\hline \multirow[t]{2}{*}{ Intestinal metaplasia } & Preoperative & 20 & $(40 \%)$ & 3 & $(6 \%)$ & - & $(-\%)$ & 23 & $(46 \%)$ & 0.157 \\
\hline & Postoperative & 15 & $(30 \%)$ & 6 & $(12 \%)$ & 1 & $(2 \%)$ & 22 & $(44 \%)$ & \\
\hline \multirow[t]{2}{*}{ H.pylori density } & Preoperative & 8 & $(16 \%)$ & 4 & $(8 \%)$ & 2 & $(4 \%)$ & 14 & $(28 \%)$ & 0.248 \\
\hline & Postoperative & 12 & $(24 \%)$ & 4 & $(8 \%)$ & 2 & $(4 \%)$ & 18 & $(36 \%)$ & \\
\hline \multirow[t]{2}{*}{ Sydney score } & Preoperative & 41 & $82(\%)$ & 8 & $(16 \%)$ & 1 & $(2 \%)$ & - & - & 0.109 \\
\hline & Postoperative & 38 & $(76 \%)$ & 10 & $(20 \%)$ & 2 & $(4 \%)$ & - & - & \\
\hline
\end{tabular}

* Wilcoxon Signed-rank test 
dyspeptic symptoms which correlated with high levels of bile refluxate and chronic gastritis on endoscopy (Wilson et al. 1995). Larusso et al. have also mentioned in a long term prospective study with ten patients that fasting bile reflux of total bile acids and DGR related gastritis increase progressively following the cholecystectomy procedure during the postoperative period (Lorusso et al. 1992). However, early onset of post-cholecystectomy DGR has presented comparable results with long-term reflux gastritis. Elhak et al. have found an increase in inactive form of chronic superficial gastritis and reflux gastritis on postoperative first year (Gad Elhak et al. 2004). Aprea et al. have evaluated elderly cholecystectomized patients with control endoscopy on postoperative 6 months and have concluded cholecystectomy is a significant risk factor for biliary gastritis (Aprea et al. 2012).

Post-cholecystectomy DGR related early chronic atrophic changes of gastric mucosa have not been clearly identified. DGR and mechanisms of gastric mucosal injury was shown in several in vitro and in vivo animal studies (Eastwood 1975; Stein et al. 1999; Nogi et al. 2001). The destructive effect of duodenal content over the gastric mucosa is defined as reactive gastritis (Sobala et al. 1993). Foveolar hyperplasia, oedema and congestion of lamina propria, acute and chronic inflammatory cells are the distinctive histopathological findings linked to reflux gastritis. Dixon et al. concluded that, prolonged exposure to the bilious duodenal content worsens mucosal injury and initiates endoscopic and histopathological sequence of gastric mucosal changes as atrophy, intestinal metaplasia and dysplasia (Dixon et al. 1986). H. pylori has been strongly accepted as a pathogen and a precursor of gastric mucosal transformation in each of these stages. Although bile reflux into the stomach is a potential inducer of intestinal metaplasia (Houghton et al. 1986), synergistic effect of the underlying $H$. pylori gastritis is stated as an essential factor in development of gastric mucosal changes (Sobala et al. 1991, 1993). The role of $H$. pylori infection in gallstones has been clearly demonstrated in several studies (Takahashi et al. 2014; Zhang et al. 2015). However, there is an ongoing debate about $H$. pylori colonization and its effects over the gastric mucosa in patients with DGR following the cholecystectomy procedure. Many studies mentioned that post-cholecystectomy DGR initiates gastric mucosal injury and reduces H. pylori colonization (Farsakh et al. 1995; Gad Elhak et al. 2004; Sobala et al. 1993; Atak et al. 2012). In contrast to these studies there are such reports demonstrated that patients with DGR after cholecystectomy have been presented with higher $H$. pylori colonization rates (Zullo et al. 1998).

In present study, early postoperative endoscopy revealed significantly higher rates of DGR and DGR related superficial chronic gastritis. Postoperative histological interpretations displayed increased $H$. pylori density, neutrophil activity, mucosal inflammation and atrophy degree. Although the Updated Sydney scores did not show significant difference between mild, moderate and severe groups, increased postoperative mean values were correlated with clinical outcomes. In this group of patients without $H$. pylori eradication therapy, postoperative pathological DGR and presence of $H$. pylori infection were found to be independent risk factors in development of early atrophic changes. These data suggested that early gastric mucosal atrophy following cholecystectomy procedure is possibly related to not only early onset of pathological DGR and excessive amount of bile refluxate but also presence of active $H$. pylori gastritis. Underlying $H$. pylori infection and DGR were thought to be the accompanying factors which potentiate the efficacy of each other's detrimental effects over the gastric mucosa.

Limitations to our study depend on the challenges in determining the postoperative development of gastritis process and exact timing of histological sampling. Most of the adult population requiring cholecystectomy procedure have concomitant gastrointestinal disorders including H. pylori gastritis and DGR. Therefore, it is difficult to identify cholecystectomy as a sole risk factor for early mucosal atrophy. As indicated in our study, defining the exclusion criteria and evaluating the cumulative effects of DGR, underlying $H$. pylori gastritis and cholecystectomy procedure over the gastric mucosa should be more effective to specify the significant postoperative outcomes. Although gastric mucosal injury advances stepwise in a long time interval, postoperative early sampling of gastric mucosa should also reveal additional information about progression of preoperative existing lesions.

In conclusion, our study demonstrated cholecystectomy as a cause of severe DGR. Early onset of pathological DGR was also strongly associated with early gastric mucosal changes. Underlying $H$. pylori infection may be the most effective factor in development of early mucosal atrophy. These independent factors influence the predisposition to DGR related chronic atrophic changes. In regard to these findings, preoperative endoscopy should be performed to reduce the additive adverse effects of $H$. pylori gastritis and DGR over the gastric mucosa by managing the appropriate medical therapy during the preoperative course.

\section{Authors' contributions}

EM, UD, DT, ED AND KS were involved in the study design and manuscript preparation, data collection, data analysis and revisions. KS performed statistical analyses, writing and manuscript preparation, EM and UD collected data and prepared study design, KS, ED and DT performed revisions of the manuscript. All authors read and approved the final manuscript. 


\section{Author details}

${ }^{1}$ Department of General Surgery, Yuksek Ihtisas Training and Research Hospital, Bursa, Turkey. ${ }^{2}$ Department of General Surgery, Bursa State Hospital, Bursa, Turkey.

\section{Acknowledgements}

This research was supported and funded by the Yuksek Ihtisas Education and Research Hospital. We thank our colleagues from General Surgery Department who performed the surgeries. We thank the anonymous referees for their useful suggestions.

\section{Competing interests}

The authors declare that they have no competing interests.

Received: 12 April 2016 Accepted: 3 November 2016

Published online: 14 November 2016

\section{References}

Aprea G et al (2012) Morpho-functional gastric pre-and post-operative changes in elderly patients undergoing laparoscopic cholecystectomy for gallstone related disease. BMC Surg 12(Suppl 1):S5

Atak l et al (2012) The effect of laparoscopic cholecystectomy on the development of alkaline reflux gastritis and intestinal metaplasia. Hepatogastroenterology 59(113):59-61

Brough WA, Taylor TV, Torrance HB (1984) The effect of cholecystectomy on duodenogastric reflux in dogs and humans. Scand J Gastroenterol Suppl 92:242-244

Buxbaum KL (1982) Bile gastritis occurring after cholecystectomy. Am J Gastroenterol 77(5):305-311

Chen $\mathrm{H}$ et al (2010) Rabeprazole combined with hydrotalcite is effective for patients with bile reflux gastritis after cholecystectomy. Can J Gastroenterol 24(3):197-201

Dixon MF et al (1986) Reflux gastritis: distinct histopathological entity? J Clin Pathol 39(5):524-530

Dixon MF et al (1996) Classification and grading of gastritis. The updated Sydney System. International workshop on the histopathology of gastritis, Houston 1994. Am J Surg Pathol 20(10):1161-1181

Dixon MF et al (2001) Bile reflux gastritis and Barrett's oesophagus: further evidence of a role for duodenogastro-oesophageal reflux? Gut 49(3):359-363

Eastwood GL (1975) Effect of pH on bile salt injury to mouse gastric mucosa. A light- and electron-microscopic study. Gastroenterology 68(6):1456-1465

Fall K, Ye W, Nyren O (2007) Risk for gastric cancer after cholecystectomy. Am J Gastroenterol 102(6):1180-1184

Farsakh NA et al (1995) Prevalence of Helicobacter pylori in patients with gall stones before and after cholecystectomy: a longitudinal study. Gut 36(5):675-678
Gad Elhak N et al (2004) Prevalence of Helicobacter pylori, gastric myoelectrical activity, gastric mucosal changes and dyspeptic symptoms before and after laparoscopic cholecystectomy. Hepatogastroenterology 51(56):485-490

Houghton PW et al (1986) Intragastric bile acids and histological changes in gastric mucosa. Br J Surg 73(5):354-356

Kellosalo J, Alavaikko M, Laitinen S (1991) Effect of biliary tract procedures on duodenogastric reflux and the gastric mucosa. Scand J Gastroenterol 26(12):1272-1278

Lin C-K et al (2009) Using the updated Sydney system to score duodenogastric reflux disease in Taiwan: the clinical value of reflux gastritis score and bile reflux index. J Med Sci 29(1):19-24

Lorusso D et al (1992) A prospective study on duodenogastric reflux and on histological changes in gastric mucosa after cholecystectomy. Gastroenterol Clin Biol 16(4):328-333

Manifold DK, Anggiansah A, Owen WJ (2000) Effect of cholecystectomy on gastroesophageal and duodenogastric reflux. Am J Gastroenterol 95(10):2746-2750

Nogi K et al (2001) Duodenogastric reflux following cholecystectomy in the dog: role of antroduodenal motor function. Aliment Pharmacol Ther 15(8):1233-1238

Perdikis G et al (1994) Altered antroduodenal motility after cholecystectomy. Am J Surg 168(6):609-614 (discussion 614-5)

Ritchie WP (1984) Alkaline reflux gastritis: a critical reappraisal. Gut 25(9):975-987

Schafer M et al (1998) Cholelithiasis—laparoscopy or laparotomy? Ther Umsch 55(2):110-115

Sobala GM et al (1991) Levels of nitrite, nitrate, N-nitroso compounds, ascorbic acid and total bile acids in gastric juice of patients with and without precancerous conditions of the stomach. Carcinogenesis 12(2):193-198

Sobala GM et al (1993) Bile reflux and intestinal metaplasia in gastric mucosa. J Clin Pathol 46(3):235-240

Stein HJ et al (1999) Bile acids as components of the duodenogastric refluxate: detection, relationship to bilirubin, mechanism of injury, and clinical relevance. Hepatogastroenterology 46(25):66-73

Takahashi Y et al (2014) Helicobacter pylori infection is positively associated with gallstones: a large-scale cross-sectional study in Japan. J Gastroenterol 49(5):882-889

Wilson P et al (1995) Pathologic duodenogastric reflux associated with persistence of symptoms after cholecystectomy. Surgery 117(4):421-428

Zhang FM et al (2015) Helicobacter pylori infection is associated with gallstones: epidemiological survey in China. World I Gastroenterol WJG 21(29):8912-8919

Zullo A et al (1998a) Gastric pathology in cholecystectomy patients: role of Helicobacter pylori and bile reflux. J Clin Gastroenterol 27(4):335-338

Zullo A et al (1998b) Gastric pathology in cholecystectomy patients: role of Helicobacter pylori and bile reflux. J Clin Gastroenterol 27(4):335-338

\section{Submit your manuscript to a SpringerOpen ${ }^{\circ}$ journal and benefit from:}

- Convenient online submission

- Rigorous peer review

- Immediate publication on acceptance

- Open access: articles freely available online

- High visibility within the field

- Retaining the copyright to your article

Submit your next manuscript at $>$ springeropen.com 